\title{
Therapeutic inhibition of CXC chemokine receptor 2 by SB225002 attenuates LPS-induced acute lung injury in mice
}

Qing $\mathrm{CaO}^{1}$, Biru Li ${ }^{1}$, Xike Wang ${ }^{2}$, Kun Sun ${ }^{3}$, Ying Guo ${ }^{2}$

\author{
${ }^{1}$ Department of Pediatrics, Shanghai Jiao Tong University Affiliated Shanghai \\ Children's Medical Center, Shanghai, China \\ 2Department of Pediatric Cardiology, Shanghai Jiao Tong University Affiliated \\ Shanghai Children's Medical Center, Shanghai, China \\ ${ }^{3}$ Department of Pediatric Cardiology, Shanghai Jiao Tong University Affiliated Xinhua \\ Hospital, Shanghai, China
}

Submitted: 7 July 2014

Accepted: 15 December 2014

Arch Med Sci 2018; 14, 3: 635-644

DOI: https://doi.org/10.5114/aoms.2017.64980

Copyright $\odot 2017$ Termedia \& Banach

\author{
Corresponding author: \\ Ying Guo \\ Department of \\ Pediatric Cardiology \\ Shanghai Jiao \\ Tong University \\ Affiliated Shanghai \\ Children's Medical Center \\ 200127 Shanghai, China \\ Phone: +8602164330607 \\ E-mail: yingguo_2014@126. \\ com
}

\begin{abstract}
Introduction: Sustained neutrophilic infiltration is known to contribute to organ damage, such as acute lung injury (ALI). CXC chemokine receptor 2 (CXCR2) is the major receptor regulating inflammatory neutrophil recruitment in acute and chronic inflamed tissues. The purpose of this study was to investigate the functional relevance of the CXCR2 inhibitor SB225002 in LPS-induced acute lung injury. Material and methods: Male C57BL/6 mice were randomly divided into the following four experimental groups ( $n=10$ per group): untreated group (control group, Ctr); LPS-treated ALI group (LPS group, LPS); LPS + PBS-treated group (LPS + PBS); and SB225002-treated ALI group (LPS + SB225002). Twenty-four hours after treatment, the blood, bronchoalveolar lavage fluid (BALF), and lung tissue were collected and wet/dry ratio, protein concentration, myeloperoxidase (MPO) activity, neutrophil infiltration, and inflammatory cytokine secretion in lung tissue were measured. The pathologic changes in the lungs were examined using optical microscopy. Survival rates were recorded at $120 \mathrm{~h}$ in all four groups, in other experiments.

Results: Histology findings revealed that the SB225002-treated group had significantly milder lung injury compared to the LPS-induced ALI and the PBS-treated control groups. Treatment with SB225002 significantly attenuated LPS-induced lung injury and suppressed the inflammatory responses in damaged lung tissue. Compared to the PBS-treated control group, treatment with SB225002 dramatically decreased the lung wet/dry ratio, protein concentration, and infiltration of neutrophils in lung tissue. Therefore, SB225002 treatment appeared to inhibit the production of inflammatory cytokines and increase survival time compared to the PBS-treated control group.

Conclusions: Together, these data demonstrated that inhibition of CXCR2 signaling by SB225002 could ameliorate LPS-induced acute lung injury, by reducing neutrophil recruitment and vascular permeability. SB225002 may be further developed as a potential novel treatment for LPS-induced ALI.
\end{abstract}

Key words: SB225002, LPS-induced ALI, CXCR2, neutrophil migration.

\section{Introduction}

Acute lung injury (ALI) and acute respiratory distress syndrome (ARDS) are clinical syndromes characterized by hypoxemia, decreased lung com- 
pliance, and bilateral pulmonary infiltrates, and which have multifactorial etiologies either from direct or indirect lung injury [1, 2]. Histopathologically, there is an initial acute exudative phase, involving alveolar-capillary leak in conjunction with leukocyte extravasation. This is followed by a fibroproliferative phase, involving the precipitation of alveolar proteins with hyaline membrane formation, persistent inflammation, and proliferation of alveolar epithelia and mesenchymal cells. Despite improvements in disease management, ALI and ARDS are still major causes of morbidity and mortality, in critically ill patients of all ages [3, 4]. The incidence of ALI/ARDS in the US is 138 in 100,000 persons per year, and is expected to double in the next 25 years [3]. Currently, the treatment of ALI/ARDS remains primarily palliative [5].

It is well known that in pulmonary inflammation, recruitment of circulating polymorphonuclear leukocytes is essential for host defense and initiates a specific immune response; a pathological hallmark of ALI and ARDS is the uncontrolled transmigration of neutrophils into the lung interstitium and alveolar space [6]. Accumulation and recruitment of pro-inflammatory leukocytes in the lung are critical events in the development of ALI [2]. The migration of leukocytes is regulated by chemotactic cytokines called chemokines. Chemokines are a large family of small chemotactic proteins divided into 4 subfamilies according to the positioning of cysteines in their primary sequences. Members with their first 2 cysteines separated by one amino acid belong to the CXC subfamily and regulate the response of polymorphonuclear (PMN) neutrophils $[7,8]$. Interleukin 8 (IL-8) is the most potent member of the CXC family and has high affinity for both of its receptors, CXCR1 and CXCR2, which are co-expressed on the membrane of neutrophils. Thus, CXCR1/2 is a major element in neutrophil recruitment [9]. Rapid neutrophil migration during inflammation, such as occurs in bacterial and fungal infections, can also be mediated by CXCR1/2-binding chemokines, elevating blood or inflammatory sites' neutrophil counts [10-12].

Depending on the underlying cause of $A L I$, different adhesion molecules and chemokines are involved in neutrophil recruitment [13]. CXCR2, the chemokine receptor for CXCL1 (keratinocyte-derived chemokine) and $\mathrm{CXCL} 2 / 3$ (macrophage inflammatory protein 2) in mice, is critically involved in neutrophil recruitment and the regulation of vascular permeability in different models of $A L I$ $[14,15]$.

In the present study, we investigated the potential beneficial effects of a potent and selective non-peptide CXCR2 antagonist, SB225002, in LPS-induced acute lung injury. We found that treatment with SB225002 ameliorated LPS-induced ALI significantly, decreasing inflammatory neutrophil infiltration and tissue damage. In addition, treatment with SB225002 also appeared to reduce MPO activity, lung wet/dry ratio, protein concentration and inflammatory cytokine production in lung tissue compared to the PBS-treated control group. Taken together, our data suggest that the potent and selective non-peptide CXCR2 antagonist SB225002 has a potential therapeutic effect on LPS-induced lung injury.

\section{Material and methods}

\section{Mice}

Forty C57BL/6 mice were purchased from the SLRC Laboratory (Shanghai, China). The mice were randomized to the following groups: control ( $n=10$, untreated), ALI ( $n=10$, LPS-treated only), PBS-treated control ( $n=10$, LPS + PBS-treated) and SB225002 ( $n=10$, SB225002-treated). Mice were sacrificed $24 \mathrm{~h}$ following LPS and group-specific treatments.

\section{Establishment of the ALI mouse model}

Female C57BL/6 mice (8-10 weeks old) were treated intraperitoneally (i.p.) with either $20 \mathrm{mg} / \mathrm{kg}$ LPS (Sigma-Aldrich, St. Louis, MO, USA) from Escherichia coli (serotype 0111:B4) in $100 \mu \mathrm{l}$ of PBS or an equal volume of PBS as a vehicle control. Mice were euthanized by an intravenous (i.v.) injection of thiopental $24 \mathrm{~h}$ following $\mathrm{ALI}$ induction and administration of group-specific treatments, the thoraxes were opened, and blood was sampled by cardiac puncture. Simultaneously, 3 bronchoalveolar lavage (BAL) procedures were performed, each with $0.5 \mathrm{ml}$ of normal saline. The fluid and blood were centrifuged ( $2000 \mathrm{~g}$, for $10 \mathrm{~min}$ ), and the supernatant and plasma were stored for further processing. Survival of mice following ALI induction and group-specific treatment was assessed and the cumulative survival curve was depicted using the Kaplan-Meier method.

\section{Histopathological analysis and lung injury scores}

Following euthanasia $24 \mathrm{~h}$ after ALI induction and administration of group-specific treatments, the whole left lobe of the lung was fixed in a $4 \%$ formaldehyde neutral buffer solution for $24 \mathrm{~h}$, dehydrated in a graded ethanol series, embedded in paraffin, and $5 \mu \mathrm{m}$ slices were cut. Paraffin sections were stained with hematoxylin-eosin $(\mathrm{H}+\mathrm{E})$ for histopathological analysis.

Severity of the lung injury was evaluated using the semi-quantitative histological index of quantitative assessment (IQA) of lung injury. Eight sec- 
tions were randomly selected from each group of mice, and 10 fields from each section were examined by microscopy at $40 \times$ magnification. A pathologist blinded to the study evaluated all the sections. The average values of lung injury obtained were considered a semi-quantitative histological IQA of lung injury (also called lung injury scores).

\section{Myeloperoxidase (MPO) activity assay}

Pulmonary neutrophil sequestration was quantified using an MPO assay as previously described [16-18]. Briefly, at the time of sacrifice, lungs were perfused with $1 \mathrm{ml}$ of $0.9 \%$ saline via the spontaneously beating right ventricle, until clear of blood. The lungs were excised and placed in $50 \mathrm{mM}$ potassium phosphate buffer solution $(\mathrm{pH}, 6.0)$ with $5 \%$ hexadecyltrimethyl ammonium bromide (Sigma-Aldrich, St. Louis, Missouri, USA), followed by homogenization and centrifugation at $15000 \mathrm{rpm}$ for $20 \mathrm{~min}$ at $4^{\circ} \mathrm{C}$. The supernatants and standard sample were added to a microtiter plate $(100 \mu \mathrm{l} /$ well) precoated with a murine anti-MPO $\mathrm{mAb}$. After incubation for $1 \mathrm{~h}$ at $37^{\circ} \mathrm{C}$, the plate was washed 6 times, followed by addition of the substrate and stop solution, after which the optical density (OD) was measured at $450 \mathrm{~nm}$ using a microplate reader. All samples were assayed in triplicate.

\section{Lung wet/dry ratio}

Lung edema during hyperoxia-induced lung injury was quantitated by determining wet : dry weight ratios as follows. A total of 10 lungs per group were ligated away from the hilum, blotted dry, weighed, and then desiccated by incubation at $130^{\circ} \mathrm{C}$ overnight in a vacuum oven. They were then reweighed to determine the dry weight, and the wet: dry ratio was then calculated.

\section{The measure of protein concentration} in lung BALF

The concentration of protein in the BALF was measured using Bradford reagent (Bio-Rad Protein Assay kit. USA). Briefly, $160 \mu \mathrm{l}$ of each standard and sample solution was pipetted into separate microtiter plate wells, and $40 \mu \mathrm{l}$ of the dye reagent was added to each well with thorough mixing. The mixture was incubated at room temperature for at least $5 \mathrm{~min}$ before the OD was measured at $595 \mathrm{~nm}$. Comparison to a standard curve provided a relative measurement of the protein concentration.

\section{Neutrophil counts}

After mice were euthanized, BALF was collected and centrifuged, and neutrophils were counted using the Kimura stain.
Intravascular and interstitial neutrophils in the lungs were distinguished by a flow cytometry-based method as previously described [19]. Briefly, an Alexa 633-labeled GR-1 antibody (clone RB6-8C5, staining kit: Invitrogen Corp., Carlsbad, CA, USA) was injected i.v. 5 min before euthanasia, labeling only intravascular neutrophils. After performing BAL, the inferior vena cava was dissected and non-adherent neutrophils were removed from the pulmonary vasculature by flushing $10 \mathrm{ml}$ of PBS at $25 \mathrm{ml} \mathrm{H}_{2} \mathrm{O}$ through the spontaneously beating right ventricle. Lungs were removed, minced, and digested with enzyme cocktail at $37^{\circ} \mathrm{C}$ for $60 \mathrm{~min}$. A cell suspension was prepared by passing the digested lungs through a $70 \mathrm{~mm}$ cell strainer (BD Falcon, Bedford, MA, USA) which lysed the erythrocytes, and the remaining leukocytes were counted. The fraction of neutrophils in the suspension was determined by flow cytometry using a FACSCalibur (Becton Dickinson, San Jose, CA, USA). Neutrophils were identified by their typical expression of CD45 (clone 30-F11, BD Biosciences-Pharmingen, San Diego, CA, USA) and GR-1 (clone RB6-8C5). The i.v. injected labeled GR-1 Ab differentiated intravascular and interstitial neutrophils.

\section{ELISA}

Tumor necrosis factor (TNF)- $\alpha$, interleukin (IL)-6, $\mathrm{IL}-1 \alpha$ and macrophage inflammatory protein (MIP)-2 amounts were detected using an ELISA according to the manufacturer's protocol. The experiment was repeated three times and results were presented as the mean.

\section{Pulmonary microvascular permeability}

Pulmonary microvascular permeability in the four experimental groups of mice was determined by Evans blue dye extravasation as described previously [15]. Briefly, Evans blue $(20 \mathrm{mg} / \mathrm{kg}$, Sigma-Aldrich) was injected i.v., $30 \mathrm{~min}$ prior to euthanasia, lungs were perfused, removed, and the Evans blue was extracted. Absorption of the dye was then measured, corrected for hemoglobin content and calculated for the different treatment groups, $24 \mathrm{~h}$ after LPS induction and group-specific treatments.

\section{Preparation of murine peripheral blood neutrophils and BM-derived PMNs}

BM from mice and BM-derived PMNs were purified from a 3-layer Percoll gradient (78\%, 66\%, and 54\%) [20]. Mouse peripheral blood neutrophils were isolated by modification of methods previously reported [21] for the purification of rabbit peripheral neutrophils. Briefly, C57BL/6 mice were volume expanded and exsanguinated into 
a $3.8 \%$ citrate solution; the blood was centrifuged at $300 \mathrm{~g}$ for $20 \mathrm{~min}$. The cell pellet was resuspended in a $6 \%$ dextran- $0.9 \% \mathrm{NaCl}$ solution $(1: 5.25)$ to a final volume of $150 \%$ of the original blood volume and sedimented at unity gravity for $30 \mathrm{~min}$. The leukocyte-rich supernatant was aspirated, washed once in Hanks' balanced salt solution (HBSS), layered on a three-step Percoll (Pharmacia) gradient $(78 \%, 66 \%$, and $54 \%)$ and centrifuged at $1060 \mathrm{~g}$ for $30 \mathrm{~min}$. Cytospin samples of the 78$66 \%$ interface revealed $90 \%$ neutrophils.

\section{Chemotaxic migration assay}

Chemotaxic migration of neutrophil and BM-derived PMNs was determined using 24-well transwell chambers (Corning, Acton, MA) with 3- $\mu \mathrm{m}$ pore size membranes as reported [22]. Briefly, isolated PMNs and neutrophils were placed on top of the filter above each well of the 24-well transwell chambers, and then the chamber was incubated for $1 \mathrm{~h}$ at $37^{\circ} \mathrm{C}$ and $5 \% \mathrm{CO}_{2}$. After incubation, the amount of migrated cells in the bottom chamber of the transwell were counted with a hemocytometer. The chemotaxic migration was expressed as the percentage of cells recovered from the bottom well compared to the total cells in the top well.

\section{Survival study in mice of LPS-induced ALI}

We determined the potential of pretreatment with SB225002 to protect against LPS-induced ALI. ALI was induced in mice with $100 \mathrm{mg} / \mathrm{kg}$ LPS in $100 \mu \mathrm{l}$ PBS by tail vein injection, then mice were randomly divided into 4 experimental groups ( $n=10$ per group) as mentioned above. Survival rates were then recorded $120 \mathrm{~h}$ following treatments.

\section{Statistical analysis}

All data were analyzed using SPSS 13.0 software and expressed as means \pm SD. Significant differences were assessed using one-way analysis of variance (ANOVA) followed by Fisher's protected least significant difference (LSD) test. A probability value of less than $0.05(p<0.05)$ was considered statistically significant.

\section{Results}

\section{SB225002 ameliorates LPS-induced ALI}

It is well known that neutrophils are essential for maintaining innate immune surveillance under normal conditions. They are also a major contributor and the first group of cells that migrate to damaged tissue during acute lung injury [23]. CXCR2 is the key chemokine receptor for neutrophils [24-26]. We therefore hypothesized that SB225002, an inhibitor of CXCR2, could alleviate the LPS-induced acute lung injury by inhibiting the infiltration of neutrophils into lung tissue. Mice with LPS-induced lung injury were treated with SB225002 via tail vein injection while PBS was the vehicle control. The results showed that lung specimens from the LPS-treated (Figure 1 B) and LPS + PBS-treated (Figure 1 C) groups displayed significant histological abnormalities, including infiltration of leukocytes into the interstitial spaces, hemorrhage, and marked swelling of the alveolar walls. Meanwhile, we discovered that treatment with SB225002 (Figure 1 D) improved the histology of the lungs compared to the PBS vehicle-treated and untreated ALI groups.

In addition, to further assess the effects of SB225002 on LPS-induced lung damage, we scored the injury in the left lungs in each group. We found that the score of the SB225002-treated ALI group was considerably lower than that of the PBS vehicle-treated and untreated ALI groups (Figure $1 \mathrm{E}, p<0.05)$.

Myeloperoxidase activity, a reliable marker of neutrophil infiltration, was also determined in differently treated lung tissue. Compared to the untreated and PBS-treated ALI groups, MPO activity was significantly $(p<0.05)$ reduced in SB225002-treated ALI mice (Figure 1 F). These results indicated that the CXCR2 inhibitory effect of SB225002 may alleviate LPS-induced morphologic lesions in lung tissue by inhibiting migration of neutrophils there.

\section{Treatment with SB225002 reduced the lung wet/dry ratio and protein concentration in lung BALF}

To quantitatively analyze the effects of SB225002 on the degree of LPS-induced lung edema index, the right upper lobe of the lungs was measured in each animal. The result showed that SB225002-treated ALI mice had a significantly lower wet/dry ratio compared to the untreated ALI and PBS-treated ALI groups (Figure $2 \mathrm{~A}$ ).

Additionally, the degree of lung injury was further assessed by determining protein concentration in lung BALF. As shown in Figure $2 \mathrm{~B}$, the concentration of protein in SB225002-treated BALF was significantly $(p<0.05)$ reduced compared to the untreated ALI and PBS-treated ALI groups. These data were consistent with the results of lung injury scores in the differently treated ALI groups.

\section{Treatment with SB225002 affected transendothelial and transepithelial migration, but not intravascular accumulation of neutrophils in lung tissue}

Following lung injury, neutrophils first accumulate in the vasculature and then infiltrate the interstitial space and exit into the BALF [27]. To 

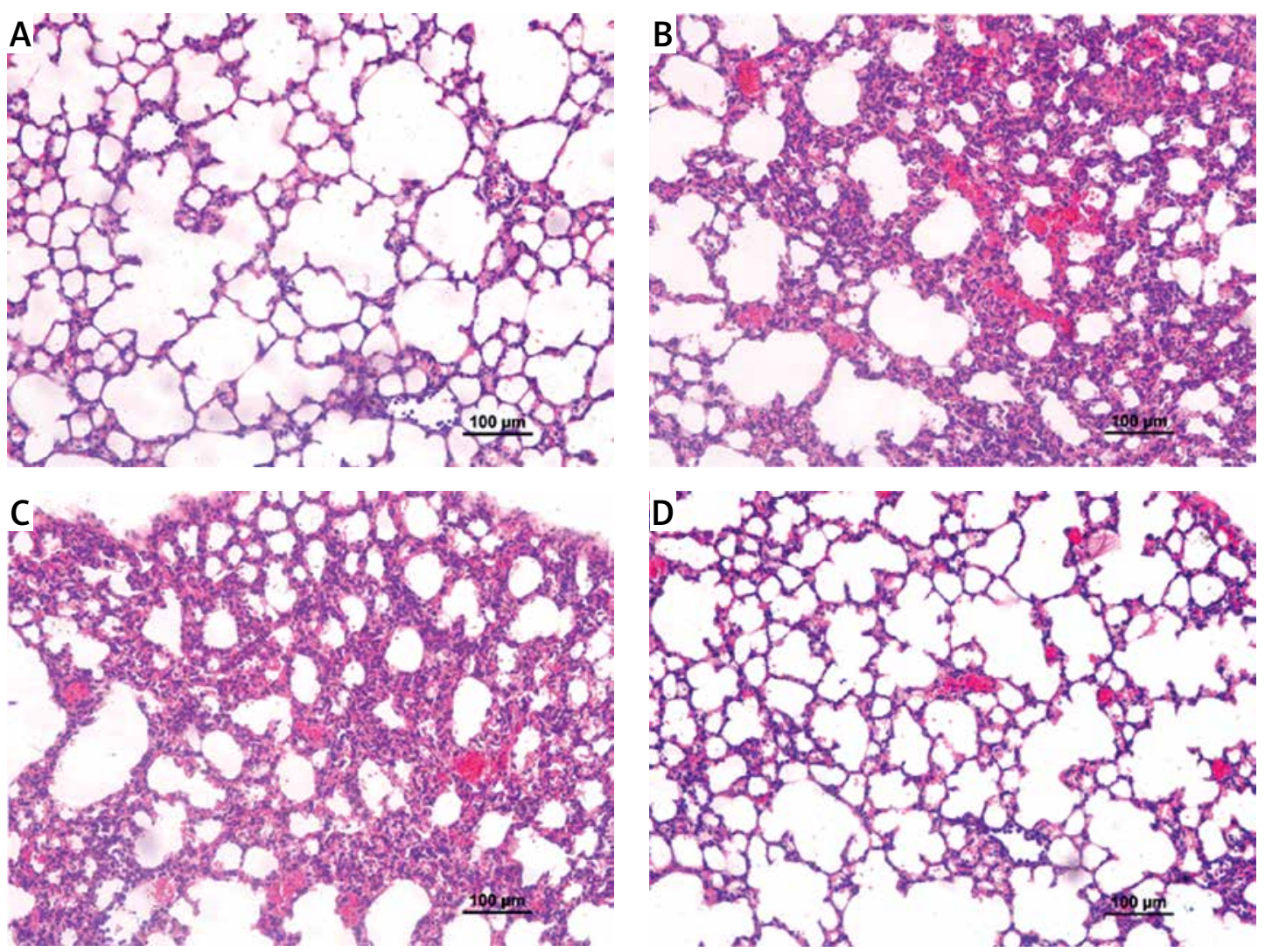

$\mathrm{E}$
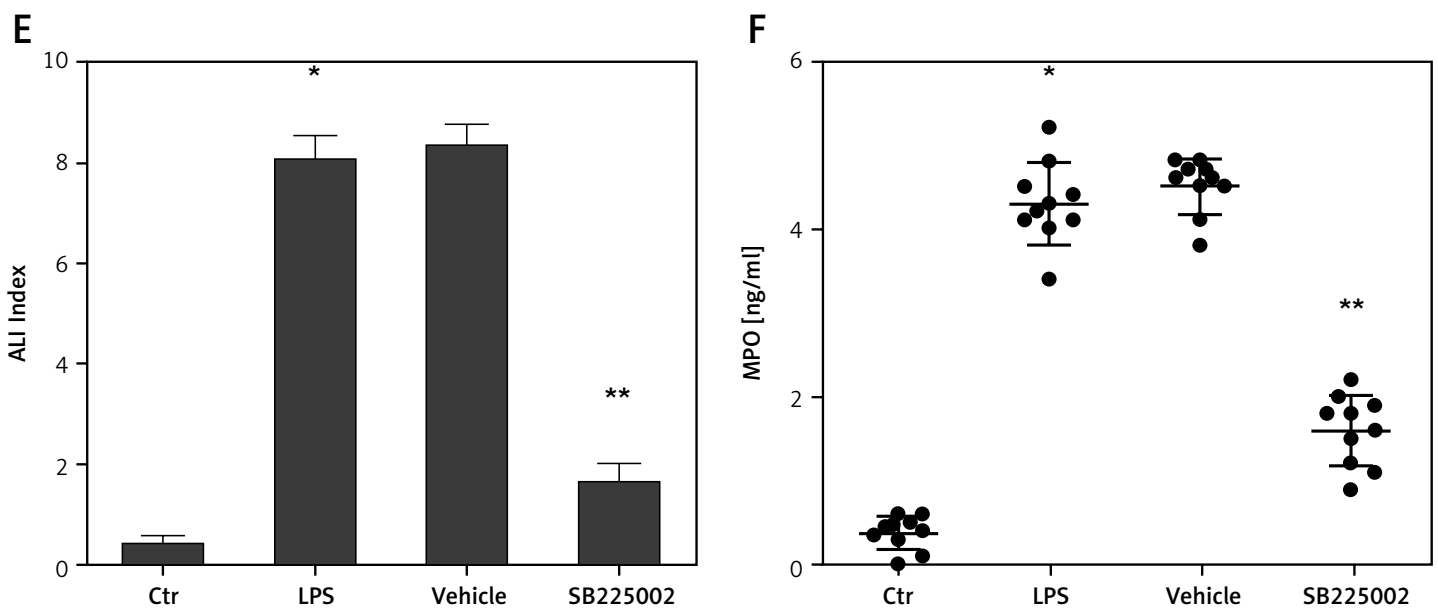

Figure 1. Histopathological index of injured murine lungs. The $\mathrm{H}+\mathrm{E}$-stained lung sections from control group (A), LPS-induced ALI group (B), vehicle-treated ALI group (C), and SB225002-treated group (D). E - The lung injury index of the differentially treated mice. F - The MPO activity in differentially treated murine lung homogenates. Data are expressed as mean \pm SEM ( $n=10$ mice per group). ${ }^{*} P<0.05$ compared to the control group; ${ }^{* *} p<0.05$ compared to the LPS-induced ALI group, or PBS-treated ALI group

ALI - acute lung injury, LPS - lipopolysaccharide, H+E - hematoxylin and eosin, Ctr - control.

determine the specific steps in the neutrophil recruitment cascade affected by SB225002-induced inhibition of CXCR2, infiltration of neutrophils into the intravascular and interstitial space and BALF was examined. We found that treatment with SB225002 significantly $(p<0.05)$ reduced the infiltration of neutrophils into the interstitial space (Figure 3 B) and BALF (Figure 3 C) compared to the untreated ALI and PBS-treated ALI groups. There was however no change in the intravascular accumulation of neutrophils (Figure $3 \mathrm{~A}$ ).

Treatment with SB225002 inhibited elevation of TNF- $\alpha$, IL-1 $\beta$, IL- 6 , and MIP-2 caused by LPS-induced lung injury

The activation and secretion of pro-inflammatory cytokines, such as tumor necrosis factor 
A

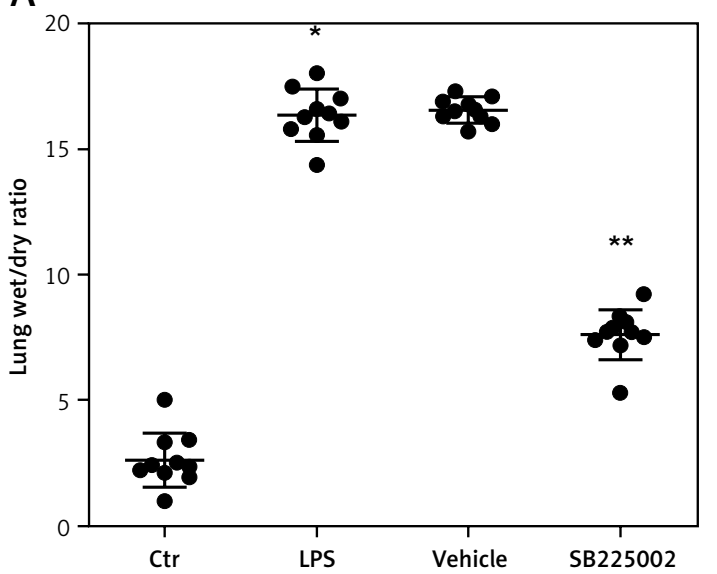

B

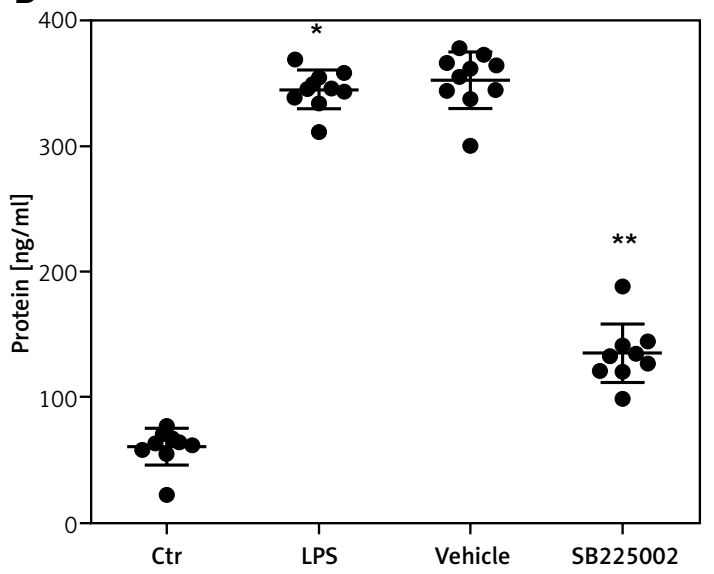

Figure 2. Lung wet/dry ratio, protein concentration in differentially treated mice BALF. A - Lung wet/dry ratio. B - Protein concentration in BALF of ALI mice following the different treatments. Data are expressed as mean \pm SEM $\left(n=10\right.$ mice per group). ${ }^{*} P<0.05$ compared to the control group; ${ }^{* \star} p<0.05$ compared to the LPS-induced ALI group or PBS-treated ALI group

ALI - acute lung injury, LPS - lipopolysaccharide, BALF - bronchoalveolar lavage fluid, Ctr - control.

(TNF)- $\alpha$, interleukin (IL)-6, IL-1 $\beta$ and macrophage inflammatory proteins (MIP)-2 in the traumatized lung, is considered to play a critical role in the pathogenesis of lung injury. To further analyze the effects of SB225002 on the inflammatory responses in LPS-induced lung injury, we determined the

A

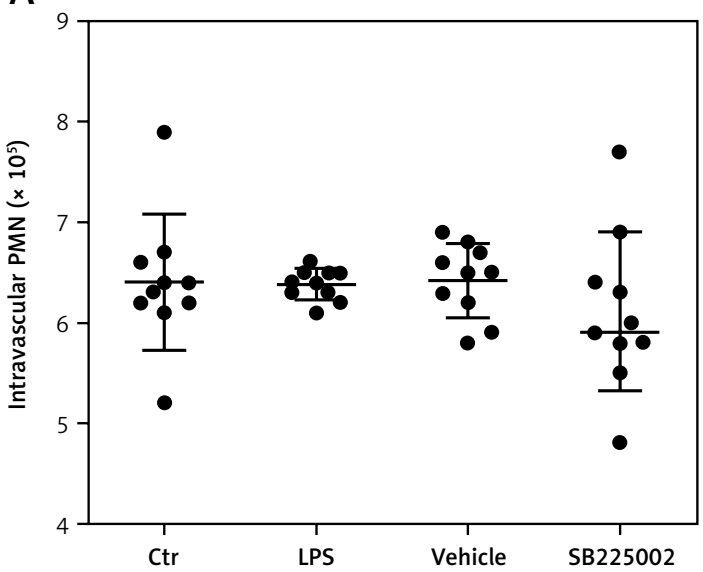

C

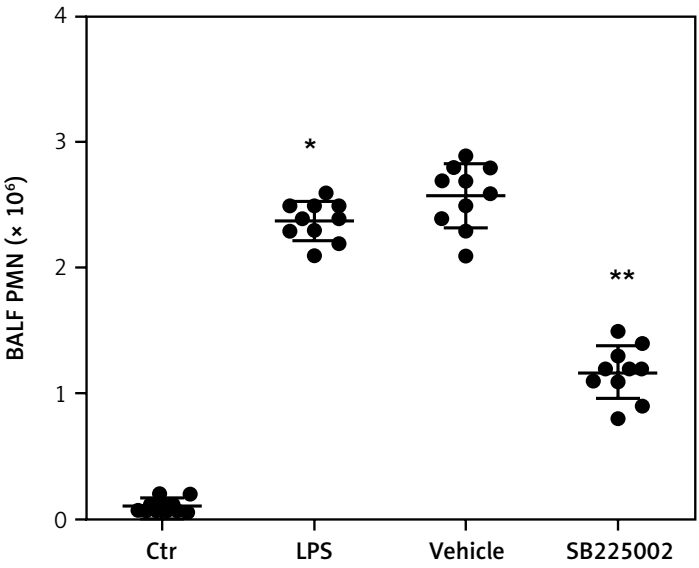

production of a number of pro-inflammatory cytokines in the BALF. We found that administration of SB225002 decreased the concentration of TNF- $\alpha$ (Figure $4 \mathrm{~A}$ ), IL-6 (Figure $4 \mathrm{~B}$ ), IL-1 $\beta$ (Figure $4 \mathrm{C}$ ), and MIP-2 (Figure $4 \mathrm{D}$ ) in BALF, compared to the untreated ALI and PBS-treated ALI groups.

B

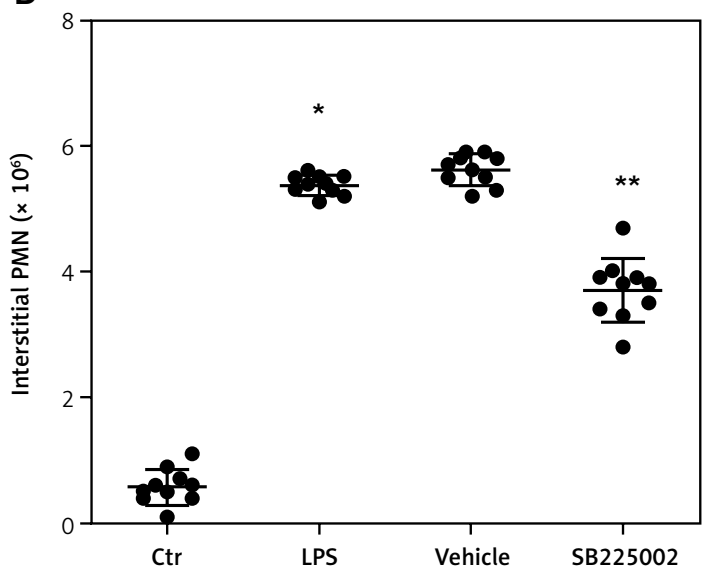

Figure 3. Neutrophil recruitment in lungs in a model of LPS-induced ALI. Neutrophil recruitment in different compartments of the lung was determined in differentially treated ALI mice $24 \mathrm{~h}$ after LPS induction. Neutrophil accumulation in the intravascular (A), interstitial (B), and alveolar (C) compartments of differentially treated mice. Data are expressed as mean \pm SEM ( $n=10$ mice per group). ${ }^{*} P<0.05$ compared to the control group mice; ${ }^{* *} p<0.05$ compared to the LPS-induced ALI group or PBS-treated ALI group

ALI - acute lung injury, LPS - lipopolysaccharide, BALF - bronchoalveolar lavage fluid, Ctr - control. 
Treatment with SB225002 reduced the pulmonary microvascular permeability

In the above experiments, we demonstrated that SB225002 suppressed the migration of neutrophils into lung tissue. In addition to neutrophil infiltration, vascular leakage is also critically involved in pulmonary inflammation and ALI. We therefore examined the effects of SB225002 on LPS-induced microvascular permeability. Determination with Evans blue (Figure 5) showed that LPS induction caused a significant increase in pulmonary vascular permeability in ALI mice, and this was attenuated by treatment with SB225002.

\section{SB225002 inhibited the migration of mice neutrophils and PMN cells in vitro}

To further quantitatively assess the relationship of CXCR2 and neutrophils or PMN cells in vitro, we investigated the relationship of CXCR2 and mice neutrophils or PMN cells and the effect of SB225002 on the migration of mice neutrophils or blood PMN cells in vitro. A 24-well chemotaxis system was loaded with chemoattractants (CXCL1, or SB225002), and the neutrophils from mice peripheral blood were placed on top of the filter above each well. As expected, compared with the negative control group (no CXCL1 and no SB225002) and positive control group (no SB225002), SB225002 inhibited the CXCL1-CXCR2 pathway-mediated chemotaxis (Figure $6 \mathrm{~A}$ ), but did not affect this process in the absence of CXCL1. Meanwhile, we observed that the migration of BM-derived PMS cells was also inhibited by SB225002 (Figure 6 B). This result was consistent with the neutrophil data in vitro. So these data provide evidence that SB225002 could impair the CXCR2-mediated migration in vitro.
A

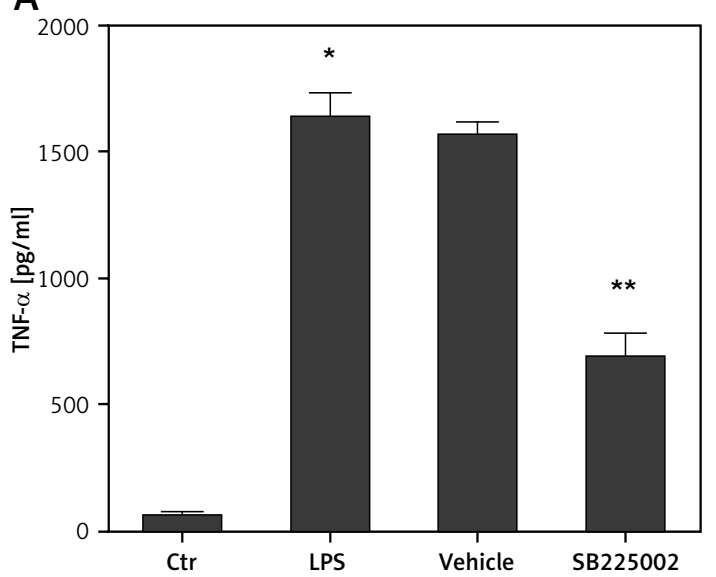

C

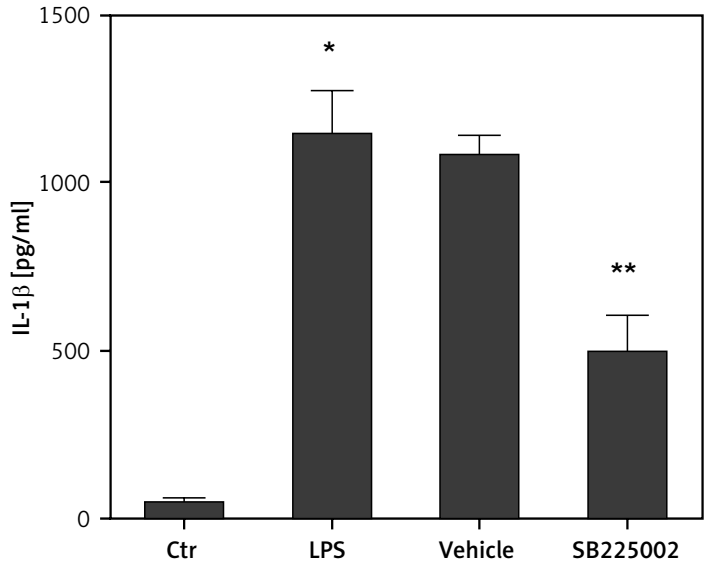

B

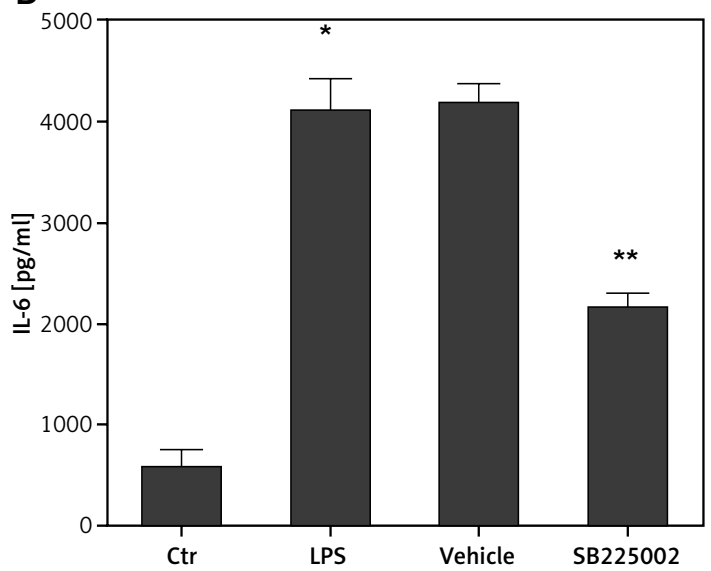

D

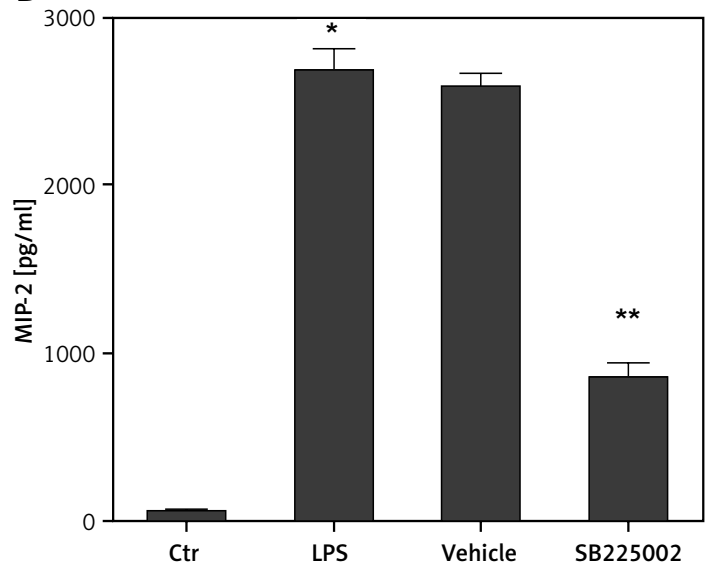

Figure 4. Production of pro-inflammatory cytokines in BALF of LPS-induced lung injury mice. Concentration of TNF- $\alpha$ (A), IL-6 (B), IL-1 $\beta$ (C) and MIP-2 (D), in BALF of mice with indicated treatment, monitored by ELISA. Data are expressed as mean \pm SEM ( $n=10$ mice per group). ${ }^{*} P<0.05$ compared to the control group mice; ${ }^{* *} p<0.05$ compared to the LPS-induced ALI group or PBS-treated ALI group

ALI - acute lung injury, LPS - lipopolysaccharide, BALF - bronchoalveolar lavage fluid, Ctr - control. 


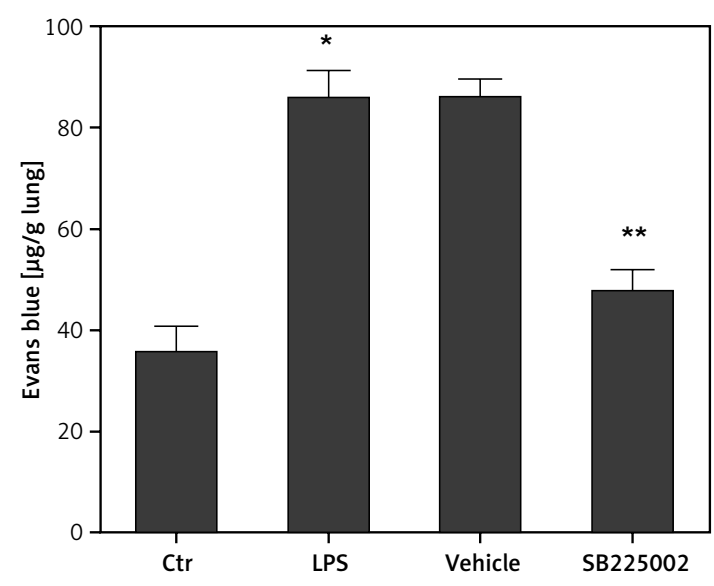

Figure 5. Effects of SB225002 on pulmonary microvascular permeability. Pulmonary permeability measured by Evans blue extravasation in differentially treated ALI mice. Data are expressed as mean \pm SEM, $n=10$ mice per group. ${ }^{*} P<0.05$ compared to the control group mice; ${ }^{* *} p<0.05$ compared to the LPS-induced ALI group or PBS-treated ALI group ALI - acute lung injury, LPS - lipopolysaccharide, Ctr-control.

\section{SB225002 improved survival of mice with LPS-induced lung injury}

To assess the long-term beneficial effects of SB225002 in LPS-induced ALI mice, we compared the survival rates between differently treated mice. Survival was significantly improved in the SB225002-treated mice compared to the untreated ALI and PBS-treated ALI groups (Figure 7). These data provide evidence supporting the therapeutic effects of SB225002 in LPS-induced ALI.

\section{Discussion}

Recent advances in the understanding of mechanisms involved in neutrophil migration have indicated an important role for the chemokine recep-

A

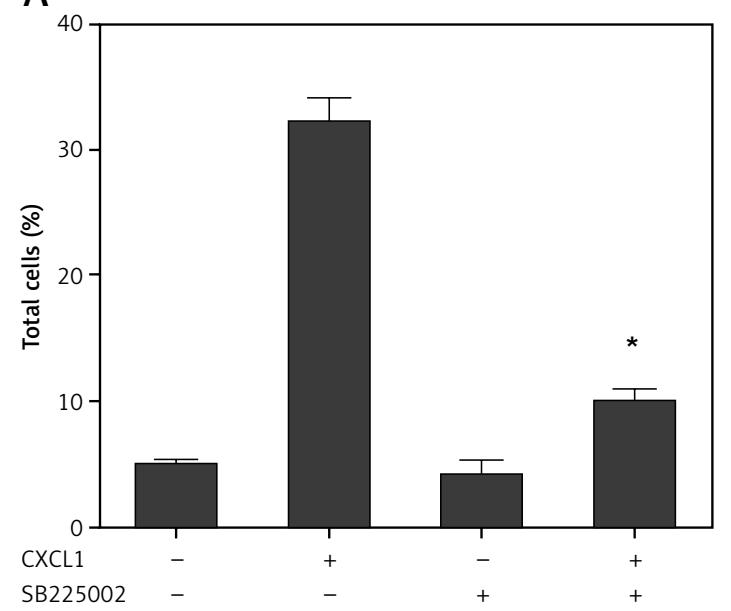

tor CXCR2 [28-30]. The infiltration of these cells mediates tissue damage and inflammatory signs in some inflammatory diseases, especially ALI [6, 31]. In the present study, we demonstrated for the first time that SB225002, a potent and selective non-peptide CXCR2 antagonist, was highly effective in ameliorating LPS-induced ALI in mice.

The ALI and ARDS are characterized by initial microvascular leakage accompanied by a neutrophil-predominant inflammatory response, which promotes diffuse alveolar damage [32-34]. It has been shown in different inflammatory models that massive infiltration of neutrophils is mediated mainly by CXCR2 activation [35, 36]. CXCR2 plays a critical role in the development of different models of ALI [14, 15, 37, 38]. Elimination of CXCR2 by gene targeting or blocking its activation with an antibody reduced neutrophil recruitment in the lung, lung edema and protein leakage [15, 37]. CXCR2 receptors on hematopoietic and non-hematopoietic cells are both essential for neutrophil recruitment in response to LPS, each accounting for about half of neutrophil recruitment [15].

Previous studies have demonstrated that blocking CXCR2 signaling attenuated ALI in different models [37, 39, 40]. Additionally, some small molecule CXCR2 antagonists have been developed as oral drugs for lung inflammation [41], including navarixin (SCH527123) [42] and SB-656933 [43]. Moreover, Leaker et al. also demonstrated that AZD8309, another CXCR2 antagonist, could inhibit the airway neutrophilic inflammation induced by LPS inhalation in human volunteers [44]. These studies suggested that a small molecule antagonist, which could inhibit CXCR2 or CXCR2-mediated inflammatory cell migration, can be used as a potential drug candidate for lung inflammatory disease. In our study, we found that treatment

B

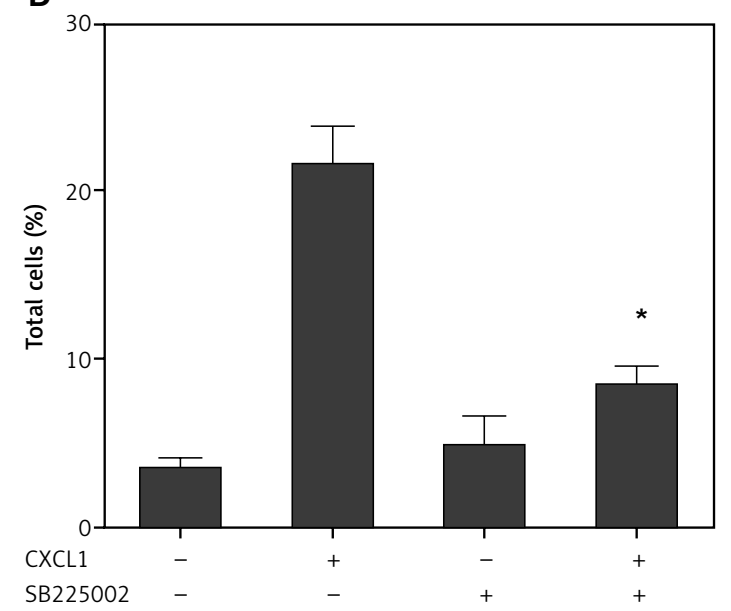

Figure 6. SB225002 inhibited the CXCR2-mediated migration of neutrophils and PMNs in vitro. The effect of SB225002 on the migration of mouse peripheral blood neutrophils (A) and BM-derived PMNs (B) in vitro. ${ }^{*} P<0.05$ compared with the positive control group (CXCL1 added and no SB225002) 
with SB225002 (Figure 1 D) improved the histology of the lung compared to the untreated $\mathrm{ALI}$ (Figure $1 \mathrm{~B}$ ) and PBS-treated groups (Figure $1 \mathrm{C}$ ). In addition, we analyzed the effects of SB225002 on the LPS-induced lung injury edema index and found that the data were similar to the results of the histological analysis (Figure $2 \mathrm{~A}$ ).

Neutrophils traveling along the endothelium can infiltrate the lung tissue and initiate a cascade of inflammatory responses, leading to endothelial damage and subsequent development of multiple organ damage [23]. Our data showed that as a marker of neutrophil influx, MPO activity was decreased in SB225002-treated mice compared to the untreated ALI and PBS-treated ALI groups (Figure $1 \mathrm{~F}$ ). Meanwhile neutrophil counts in different parts of lung tissue showed that SB225002 did not affect the intravascular neutrophil count (Figure $3 \mathrm{~A}$ ), but significantly reduced neutrophils in the interstitial space (Figure 3 B) and BALF (Figure 3 C). To further confirm the beneficial effect of SB225002 on lung injury, we measured the protein concentration in BALF. Our results showed that treatment of LPS-induced ALI mice with SB225002 reduced protein concentration in lung BALF (Figure 2 B).

In addition, inflammatory responses play an important role in the development and pathogenesis of LPS-induced lung injury. We therefore determined the production of inflammatory cytokines in the lung BALF and observed that treatment with SB225002 dramatically reduced secretion of TNF- $\alpha$ (Figure 4 A), IL-6 (Figure 4 B), IL-1 $\beta$ (Figure $4 \mathrm{C}$ ) and MIP-2 (Figure $4 \mathrm{D}$ ). These data indicate that SB225002 may impair the inflammatory responses through inhibition of inflammatory cell migration.

In conclusion, our data suggest that SB225002 may be potentially beneficial in the therapy of ALI. SB225002 treatment improved the histology of lung tissue, reduced neutrophil infiltration, and decreased secretion of pro-inflammatory cytokines in the process of lung injury. It may therefore provide a novel drug candidate for the treatment of LPS-induced lung injury via inhibition of migration of neutrophils from the blood to damaged lung tissue.

\section{Acknowledgments}

Qing Cao and Biru Li are co-first authors.

This work was financially supported by the Pudong New Area science and technology development foundation innovation fund (PKJ2011-Y32), China National Funds for Young Scientists (81201450) and SCMC-YZ1012. The authors thank Shanghai Jiao Tong University Affiliated Shanghai Children's Medical Center for technical assistance.

\section{Conflict of interest}

The authors declare no conflict of interest.

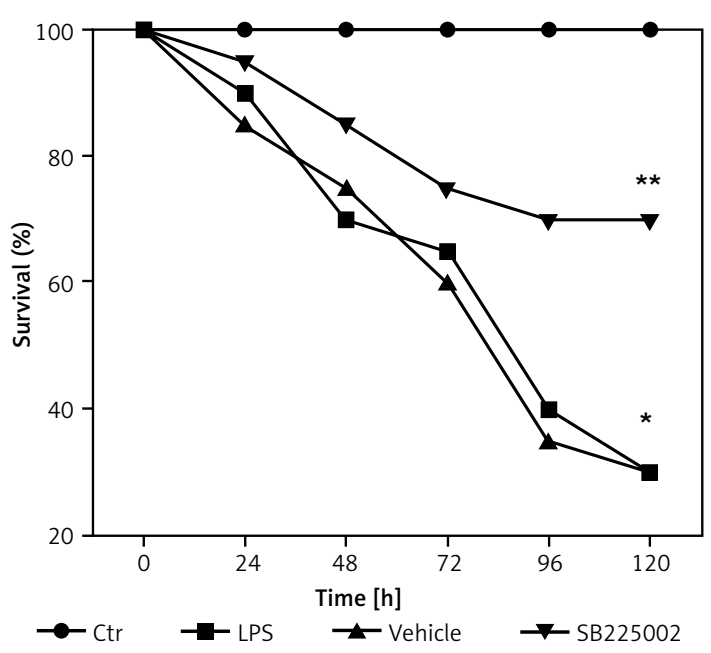

Figure 7. Treatment with SB225002 prolonged the survival of mice with LPS-induced lung injury. The Kaplan-Meier survival curves of mice $(n=10)$ with indicated treatments were monitored. ${ }^{*} P<0.05$ compared to the control group; ${ }^{* *} p<0.05$ compared to the LPS-induced ALI group mice or PBS-treated ALI group

ALI - acute lung injury, LPS - lipopolysaccharide, Ctr control.

\section{References}

1. Matthay MA, Ware LB, Zimmerman GA. The acute respiratory distress syndrome. J Clin Invest 2012; 122: 2731-40.

2. Ware LB, Matthay MA. The acute respiratory distress syndrome. N Engl J Med 2000; 342: 1334-49.

3. Rubenfeld GD, Caldwell E, Peabody E, et al. Incidence and outcomes of acute lung injury. N Engl J Med 2005; 353: 1685-93.

4. Erickson SE, Martin GS, Davis JL, Matthay MA, Eisner MD. Recent trends in acute lung injury mortality: 19962005. Crit Care Med 2009; 37: 1574-9.

5. Matthay MA, Zimmerman GA, Esmon C, et al. Future research directions in acute lung injury: summary of a $\mathrm{Na}$ tional Heart, Lung, and Blood Institute working group. Am J Respir Crit Care Med 2003; 167: 1027-35.

6. Martin TR. Neutrophils and lung injury: getting it right. J Clin Invest 2002; 110: 1603-5.

7. Baggiolini M. Chemokines in pathology and medicine. J Intern Med 2001; 250: 91-104.

8. Dumont RA, Car BD, Voitenok NN, et al. Systemic neutralization of interleukin-8 markedly reduces neutrophilic pleocytosis during experimental lipopolysaccharide-induced meningitis in rabbits. Infect Immun 2000; 68:5756-63.

9. Murphy PM. Neutrophil receptors for interleukin-8 and related CXC chemokines. Semin Hematol 1997; 34: 311-8.

10. Stillie R, Farooq SM, Gordon JR, Stadnyk AW. The functional significance behind expressing two IL-8 receptor types on PMN. J Leukoc Biol 2009; 86: 529-43.

11. Christopher MJ, Link DC. Regulation of neutrophil homeostasis. Curr Opin Hematol 2007; 14: 3-8.

12. von Vietinghoff $S$, Ley K. Homeostatic regulation of blood neutrophil counts. J Immunol 2008; 181: 5183-8.

13. Doerschuk CM. Mechanisms of leukocyte sequestration in inflamed lungs. Microcirculation 2001; 8: 71-88.

14. Belperio JA, Keane MP, Burdick MD, et al. Critical role for CXCR2 and CXCR2 ligands during the pathogenesis of 
ventilator-induced lung injury. J Clin Invest 2002; 110: 1703-16.

15. Reutershan J, Morris MA, Burcin TL, et al. Critical role of endothelial CXCR2 in LPS-induced neutrophil migration into the lung. J Clin Invest 2006; 116: 695-702.

16. Keane MP, Belperio JA, Moore TA, et al. Neutralization of the CXC chemokine, macrophage inflammatory protein-2, attenuates bleomycin-induced pulmonary fibrosis. J Immunol 1999; 162: 5511-8.

17. Colletti LM, Kunkel SL, Walz A, et al. Chemokine expression during hepatic ischemia/reperfusion-induced lung injury in the rat. The role of epithelial neutrophil activating protein. J Clin Invest 1995; 95: 134-41.

18. Gray KD, Simovic MO, Chapman WC, et al. Endotoxin potentiates lung injury in cerulein-induced pancreatitis. Am J Surg 2003; 186: 526-30.

19. Zarbock A, Singbartl K, Ley K. Complete reversal of acid-induced acute lung injury by blocking of platelet-neutrophil aggregation. J Clin Invest 2006; 116: 3211-9.

20. Suratt BT, Young SK, Lieber J, Nick JA, Henson PM, Worthen GS. Neutrophil maturation and activation determine anatomic site of clearance from circulation. Am J Physio Lung Cell Mol Physiol 2001; 281: L913-21.

21. Haslett C, Worthen GS, Giclas PC, Morrison DC, Henson JE, Henson PM. The pulmonary vascular sequestration of neutrophils in endotoxemia is initiated by an effect of endotoxin on the neutrophil in the rabbit. Am Rev Respir Dis 1987; 136: 9-18.

22. Farooq SM, Stillie R, Svensson M, Svanborg C, Strieter RM, Stadnyk AW. Therapeutic effect of blocking CXCR2 on neutrophil recruitment and dextran sodium sulfate-induced colitis. J Pharmacol Exp Ther 2009; 329: 123-9.

23. Grommes J, Soehnlein O. Contribution of neutrophils to acute lung injury. Mol Med 2011; 17: 293-307.

24. Ahuja SK, Lee JC, Murphy PM. CXC chemokines bind to unique sets of selectivity determinants that can function independently and are broadly distributed on multiple domains of human interleukin-8 receptor B. Determinants of high affinity binding and receptor activation are distinct. J Biol Chem 1996; 271: 225-32.

25. Holmes WE, Lee J, Kuang WJ, Rice GC, Wood WI. Structure and functional expression of a human interleukin-8 receptor. Science 1991; 253: 1278-80.

26. Murphy PM, Tiffany HL. Cloning of complementary DNA encoding a functional human interleukin-8 receptor. Science 1991; 253: 1280-3.

27. Reutershan J, Basit A, Galkina EV, Ley K. Sequential recruitment of neutrophils into lung and bronchoalveolar lavage fluid in LPS-induced acute lung injury. Am J Physiol Lung Cell Mol Physiol 2005; 289: L807-15.

28. Liu L, Li M, Spangler LC, et al. Functional defect of peripheral neutrophils in mice with induced deletion of CXCR2. Genesis 2013; 51: 587-95.

29. Eash KJ, Greenbaum AM, Gopalan PK, Link DC. CXCR2 and CXCR4 antagonistically regulate neutrophil trafficking from murine bone marrow. J Clin Invest 2010; 120: 2423-31.

30. Wu Y, Wang S, Farooq SM, et al. A chemokine receptor CXCR2 macromolecular complex regulates neutrophi functions in inflammatory diseases. J Biol Chem 2012; 287: 5744-55.

31. Abraham E. Neutrophils and acute lung injury. Crit Care Med 2003; 31 (4 Suppl.): S195-9.

32. Azoulay E, Lemiale V, Mokart D, et al. Acute respiratory distress syndrome in patients with malignancies. Intensive Care Med 2014; 40: 1106-14.
33. de Luis Cabezon N, Sanchez Castro I, Bengoetxea Uriarte UX, Rodrigo Casanova MP, Garcia Pena JM, Aguilera Celorrio L. Acute respiratory distress syndrome: A review of the Berlin definition [Spanish]. Rev Esp Anestesiol Reanim 2014; 61: 319-27.

34. Walter JM, Wilson J, Ware LB. Biomarkers in acute respiratory distress syndrome: from pathobiology to improving patient care. Expert Rev Respir Med 2014; 8 : 573-86.

35. Buanne P, Di Carlo E, Caputi L, et al. Crucial pathophysiological role of CXCR2 in experimental ulcerative colitis in mice. J Leukoc Biol 2007; 82: 1239-46.

36. White JR, Lee JM, Young PR, et al. Identification of a potent, selective non-peptide CXCR2 antagonist that inhibits interleukin-8-induced neutrophil migration. J Biol Chem 1998; 273: 10095-8.

37. Sue RD, Belperio JA, Burdick MD, et al. CXCR2 is critical to hyperoxia-induced lung injury. J Immunol 2004; 172 3860-8.

38. Strieter RM, Keane MP, Burdick MD, Sakkour A, Murray LA, Belperio JA. The role of CXCR2/CXCR2 ligands in acute lung injury. Curr Drug Targets Inflamm Allergy 2005; 4: 299-303.

39. Zarbock A, Allegretti M, Ley K. Therapeutic inhibition of CXCR2 by reparixin attenuates acute lung injury in mice. Br J Pharmacol 2008; 155: 357-64.

40. Lomas-Neira JL, Chung CS, Grutkoski PS, Miller EJ, Ayala A. CXCR2 inhibition suppresses hemorrhage-induced priming for acute lung injury in mice. J Leukoc Biol 2004; 76: 58-64.

41. Donnelly LE, Barnes PJ. Chemokine receptor CXCR2 antagonism to prevent airway inflammation. Drugs Future 2011; 36: 465-72.

42. Holz O, Khalilieh S, Ludwig-Sengpiel A, et al. SCH527123, a novel CXCR2 antagonist, inhibits ozone-induced neutrophilia in healthy subjects. Eur Respir J 2010; 35 564-70.

43. Lazaar AL, Sweeney LE, MacDonald AJ, Alexis NE, Chen C Tal-Singer R. SB-656933, a novel CXCR2 selective antagonist, inhibits ex vivo neutrophil activation and ozone-induced airway inflammation in humans. $\mathrm{Br}$ J Clin Pharmacol 2011; 72: 282-93.

44. Leaker BR, Barnes PJ, O'Connor B. Inhibition of LPS-induced airway neutrophilic inflammation in healthy volunteers with an oral CXCR2 antagonist. Respir Res 2013; 14: 137. 